\title{
Resveratrol regulates autophagy signaling in chronically ischemic myocardium
}

\author{
Ashraf A. Sabe, MD, Nassrene Y. Elmadhun, MD, Rahul S. Dalal, BS, Michael P. Robich, MD, and \\ Frank W. Sellke, MD
}

Objective: Autophagy is a cellular process by which damaged components are removed. Although autophagy can result in cell death, when optimally regulated, it might be cardioprotective. Resveratrol is a naturally occurring polyphenol also believed to be cardioprotective. Using a clinically relevant swine model of metabolic syndrome, we investigated the effects of resveratrol on autophagy in the chronically ischemic myocardium.

\begin{abstract}
Methods: Yorkshire swine were fed a regular diet $(n=7)$, a high cholesterol diet $(n=7)$, or a high cholesterol diet with supplemental resveratrol $(n=6)$. After 4 weeks, an ameroid constrictor was surgically placed on the left circumflex artery to induce chronic myocardial ischemia. The diets were continued another 7 weeks, and then the ischemic and nonischemic myocardium were harvested for protein analysis.

Results: In the ischemic myocardium, a high cholesterol diet partly attenuated the autophagy, as determined by an increase in phosphorylated mammalian target of rapamycin (p-mTOR) and a decrease in p70 S6 kinase (P70S6K), lysosome-associated membrane protein (LAMP)-2, and autophagy-related gene 12-5 conjugate (ATG 12-5; $P<.05$ ). The addition of resveratrol blunted many of these changes, because the p-mTOR, P70S6K, and LAMP-2 levels were not significantly altered from those of the pigs fed a regular diet. Other autophagy markers were increased with a high cholesterol diet, including light chain 3A-II and beclin $1(P<.05)$. In the nonischemic myocardium, beclin 1 was decreased in the high cholesterol-fed pigs $(P<.05)$; otherwise no significant changes in protein expression were noted among the 3 groups.
\end{abstract}

Conclusions: In the chronically ischemic myocardium, resveratrol partly reversed the effects of a high cholesterol diet on autophagy. This might be a mechanism by which resveratrol exerts its cardioprotective effects. (J Thorac Cardiovasc Surg 2014;147:792-9)

Autophagy is an adaptive process by which damaged cellular components are removed or recycled. When optimally regulated, autophagy is cardioprotective during myocardial stress. ${ }^{1-4}$ When cardiac myocytes are hypoxic or deprived of nutrients, autophagy will be upregulated, and in the setting of caloric excess and metabolic syndrome, autophagy will be diminished. ${ }^{3}$ With the continued increase in the incidence of obesity and diabetes, cardiovascular disease remains the leading cause of death in

From the Division of Cardiothoracic Surgery, Cardiovascular Research Center, Brown University Warren Alpert School of Medicine, Providence, RI.

The present study was supported by the National Heart, Lung, and Blood Institute (grants R01HL46716, R01HL69024, and R01HL85647, to Dr Sellke), National Institutes of Health training grant 5T32-HL094300-03 (to Drs Sabe and Elmadhun), and National Institutes of Health training grant T32-HL0074 (to Dr Robich).

Disclosures: Dr Sabe reports consulting fees from the Medicines Company and CSL Behring. All other authors have nothing to disclose with regard to commercial support.

Read at the 93rd Annual Meeting of The American Association for Thoracic Surgery, Minneapolis, Minnesota, May 4-8, 2013.

Received for publication April 3, 2013; revisions received June 6, 2013; accepted for publication June 18, 2013; available ahead of print Nov 22, 2013.

Address for reprints: Frank W. Sellke, MD, Division of Cardiothoracic Surgery, Cardiovascular Research Center, Brown University Warren Alpert School of Medicine, 2 Dudley St, MOC 360, Providence, RI 02905 (E-mail: fsellke@ lifespan.org).

$0022-5223 / \$ 36.00$

Copyright (C) 2014 by The American Association for Thoracic Surgery

http://dx.doi.org/10.1016/j.jtcvs.2013.06.062 the developed world. ${ }^{5,6}$ Metabolic syndrome not only increases the risk of coronary artery disease but also results in increased morbidity and mortality after ischemic injury. ${ }^{7,8}$ In cardiac surgery patients, impaired autophagy has been shown to result in unfavorable remodeling and development of postoperative atrial fibrillation.,10 Autophagy flux during cardiac surgery has also been shown to correlate with surgical risk scores and the crossclamp time. ${ }^{11}$ Novel mechanisms to regulate autophagy can lead to a reduction of surgical risk and might improve the operative outcomes.

In animal models, resveratrol supplementation has been shown to mimic caloric restriction and to induce autophagy. ${ }^{12,13}$ This naturally occurring polyphenol, found in many berries, as well as in the grapes used to make red wine, is believed to be cardioprotective in chronically ischemic myocardium. ${ }^{14}$ In France, despite a diet high in saturated fats, the mortality rates from cardiovascular disease has been shown to be lower than in other countries. This phenomenon, termed the "French Paradox," is believed to be related to the greater consumption of red wine and has sparked interest in the potential benefits of resveratrol. ${ }^{15}$

The reported advantages of resveratrol are vast and include increased longevity and benefits against cancer, inflammation, and cardiovascular disease. The mechanism 


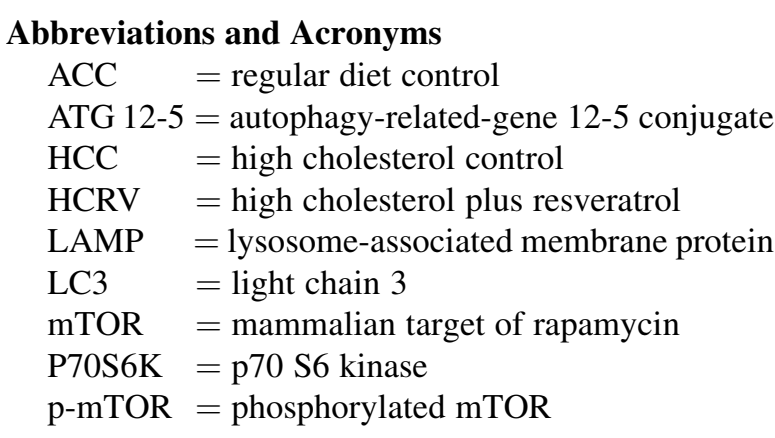

by which resveratrol exerts its cardioprotective effects remains under investigation.

In a swine model of metabolic syndrome and chronic myocardial ischemia, we have previously demonstrated that resveratrol prevented regional wall motion dysfunction and preserved endothelium-dependent coronary function and myocardial perfusion. ${ }^{14}$ However, no increase was found in collateral formation, and the antiangiogenic markers were actually increased. ${ }^{16}$ Using this model, we have also demonstrated that in peripheral tissues resveratrol improved inflammation and insulin and glucose signaling. ${ }^{17,18}$ However, in the ischemic myocardium, insulin signaling remained largely unchanged. $^{19}$ In rodents, resveratrol has been shown to prevent harmful postinfarction remodeling through the induction of autophagy. ${ }^{12,13}$ These results have suggested that resveratrol acts on alternate pathways in a tissue-specific manner. In the ischemic myocardium, regulation of autophagy might be a potential mechanism by which resveratrol supplementation prevents endothelial dysfunction and cardiac remodeling. Thus, using a clinically relevant swine model of metabolic syndrome, we sought to investigate the effects of resveratrol on autophagy in chronic myocardial ischemia.

\section{METHODS}

\section{Animal Model and Surgical Interventions}

Adult male Yorkshire miniswine (Parsons Research, Amherst, Mass) were divided into 3 groups according to the daily diet fed during an 11-week period. The regular diet control (ACC) group was given no drug and was fed a regular chow diet $(n=7)$. The high cholesterol control (HCC) group $(\mathrm{n}=7)$ was given daily feedings of $500 \mathrm{~g}$ of a high cholesterol diet providing $2248 \mathrm{kcal} / \mathrm{d}$ consisting of $75 \%$ regular chow, $4 \%$ cholesterol, $17.2 \%$ coconut oil, $2.3 \%$ corn oil, and $1.5 \%$ sodium cholate. The third group was fed the same diet as the HCC group but was also supplemented with daily oral resveratrol, $100 \mathrm{mg} / \mathrm{kg} / \mathrm{d}$ (HCRV group, $\mathrm{n}=6$; ChromaDex, Irvine, Calif). The feedings were observed to confirm complete consumption of the food and supplement. After 4 weeks of dietary adherence, the pigs in both study groups (HCC and HCRV groups) underwent surgical placement of a titanium (1.75-2.25 $\mathrm{mm}$ in diameter) ameroid constrictor (Research Instruments SW, Escondito, Calif) on the proximal left circumflex coronary artery.

The anesthesia used for the procedures and the perioperative analgesia and antibiotic prophylaxis have been previously described. ${ }^{14}$ The methods for myocardial perfusion analysis and coronary angiography have also been previously described in these pigs. ${ }^{14}$

At 7 weeks after ameroid placement, the pigs were killed, and their hearts were harvested. Tissue samples from the chronically ischemic myocardium in the left circumflex coronary artery territory and nonischemic myocardium were rapidly frozen in liquid nitrogen for molecular studies. The Institutional Animal Care and Use Committee of the Rhode Island Hospital approved all the experiments. The pigs were cared for in compliance with the "Principles of Laboratory Animal Care" formulated by the National Society for Medical Research and the "Guide for the Care and Use of Laboratory Animals."

\section{Western Blots}

Radioimmunoprecipitation assay buffer (Boston BioProducts, Worcester, Mass) or $2 \%$ sodium dodecyl sulfate-based detergent was used to isolate whole cell lysates from homogenized tissue. The myocardial samples from the chronically ischemic territories and nonischemic territories were assessed separately. The total protein concentration was determined using the bicinchoninic acid assay (Pierce, Thermo Scientific, Rockford, Ill). A total of $40 \mu \mathrm{g}$ of total protein was fractionated using $4 \%$ to $12 \%$ Bis-Tris gel electrophoresis (NuPage Novex Mini Gel, Invitrogen, Carlsbad, Calif). The protein samples were transferred to polyvinylidene difluoride membranes (Millipore, Bedford, Mass). Each membrane was incubated overnight at $4{ }^{\circ} \mathrm{C}$ with the following primary antibodies: mammalian target of rapamycin (mTOR), phosphorylated mTOR (p-mTOR), p70 S6 kinase (P70S6K), beclin 1, autophagy-related-gene 12-5 conjugate (ATG12-5), light chain 3 (LC3)A-II, LC3B-II, and lysosome-associated membrane protein (LAMP)-1 (all from Cell Signaling Technology, Danvers, Mass) and LAMP-2 (Life Technologies, Grand Island, NY). The lysates made in radioimmunoprecipitation assay buffer were used for Western blotting of mTOR and p-mTOR. All the other primary antibodies were incubated on membranes prepared with lysates isolated in sodium dodecyl sulfate. The membranes were incubated with the appropriate horseradish peroxidase-linked secondary antibody at room temperature for approximately 1 hour (Jackson ImmunoResearch, West Grove, Pa). The immune complexes were visualized using enhanced chemiluminescence, and the images were captured with a digital camera system (G-Box Syngene, Cambridge, UK). All membranes were probed with glyceraldehyde-3-phosphate dehydrogenase (Cell Signaling Technology) to correct for protein loading.

\section{Statistical Analysis}

The Western blot bands were quantified with densitometry using Image-J software (National Institutes of Health, Bethesda, Md). The probability values for the Western blot data are reported from 2-way analysis of variance followed by a post hoc Bonferroni test of the densitometry arbitrary units. The Western blot results are expressed as the $\mathrm{x}$-fold change \pm standard error of the mean compared with the ACC group (Tables 1 and 2 and Figure 2).

\section{RESULTS}

\section{Animal Model}

All the pigs included for analysis survived the study to completion. As previously reported, ${ }^{14} 7$ weeks after ameroid placement, no change was found in the myocardial blood flow at rest. However, when stressed with ventricular pacing, the adjusted myocardial blood flow in the ischemic area was improved in the HCRV group compared with the HCC group. ${ }^{14}$ Also, 7 weeks after placement of the ameroid constrictor, coronary catheterization demonstrated that all the pigs had $99 \%$ to $100 \%$ occlusion of the left circumflex coronary artery. ${ }^{14}$ 
TABLE 1. Protein expression in ischemic myocardium

\begin{tabular}{lccrl}
\hline \multicolumn{1}{c}{ Target } & ACC group & HCC group & HCRV group & $\boldsymbol{P}$ value \\
\hline mTOR & $1 \pm 0.252$ & $2.09 \pm 0.534$ & $1 \pm 0.207$ & .083 \\
p-mTOR & $1 \pm 0.218$ & $4.09 \pm 0.555$ & $2.19 \pm 0.560$ & $.0006^{*}, \dagger$ \\
P70S6K & $1 \pm 0.279$ & $0.25 \pm 0.062$ & $0.40 \pm 0.123$ & $.017^{*}$ \\
Beclin 1 & $1 \pm 0.257$ & $2.55 \pm 0.408$ & $2.66 \pm 0.435$ & $.0083^{*}$, \\
ATG 12-5 & $1 \pm 0.353$ & $0.12 \pm 0.063$ & $0.06 \pm 0.015$ & $.0028^{*}, \ddagger$ \\
LAMP-1 & $1 \pm 0.306$ & $1.24 \pm 0.182$ & $0.74 \pm 0.138$ & .34 \\
LAMP-2 & $1 \pm 0.248$ & $0.26 \pm 0.041$ & $0.43 \pm 0.129$ & $.013^{*}$ \\
LC3A-II & $1 \pm 0.087$ & $3.06 \pm 0.725$ & $1.74 \pm 0.274$ & $.017^{*}$ \\
LC3B-II & $1 \pm 0.158$ & $0.96 \pm 0.238$ & $1.62 \pm 0.488$ & .28 \\
\hline
\end{tabular}

Protein expression listed as $\mathrm{x}$-fold change \pm standard error of the mean compared with the ACC group. One-way analysis of variance performed to determine the $P$ value. Bonferroni multiple comparison tests were performed. $A C C$, Regular diet control; HCC, high cholesterol control; HCRV, high cholesterol plus resveratrol; mTOR, mammalian target of rapamycin; $p$-mTOR, phosphorylated mTOR; $P 70 S 6 K$, p70 S6 kinase; ATG 12-5, autophagy-related gene 12-5 conjugate; LAMP, lysosome-associated membrane protein; $L C 3$, light chain 3. *Significant difference between ACC and HCC groups. †Significant difference between HCC and HCRV groups. †़Significant difference between ACC and HCRV groups.

The body mass index for each pig was calculated after initiation of the diets at 4 and 11 weeks. No significant differences were found in the body mass index among the pigs in all 3 groups after 4 weeks (Figure 1). After 11 weeks, however, the body mass index was significantly higher in the HCC group than in either the ACC group or HCRV group $(P=.0042$; Figure 1). After 11 weeks, $50 \mathrm{~g}$ of dextrose was administered intravenously. The blood glucose levels measured 30 minutes after the dextrose infusion were significantly greater in the HCC group than in either the ACC or HCRV group $(P=.014$; Figure 1$)$.

\section{Western Blots}

In the ischemic myocardium, the pigs given a high cholesterol diet alone (HCC group) demonstrated a fourfold increase in p-mTOR and a decrease in P70S6K compared with the pigs fed a regular diet (ACC group; $P=.0006$

TABLE 2. Protein expression in nonischemic myocardium

\begin{tabular}{lcccc}
\hline \multicolumn{1}{c}{ Target } & ACC group & HCC group & HCRV group & $\boldsymbol{P}$ value \\
\hline mTOR & $1 \pm 0.154$ & $1.64 \pm 0.272$ & $0.94 \pm 0.383$ & .16 \\
p-mTOR & $1 \pm 0.172$ & $1.82 \pm 0.478$ & $0.92 \pm 0.100$ & .22 \\
P70S6K & $1 \pm 0.199$ & $1.05 \pm 0.112$ & $1.03 \pm 0.170$ & .98 \\
Beclin 1 & $1 \pm 0.163$ & $0.56 \pm 0.039$ & $0.75 \pm 0.068$ & $.0098^{*}$ \\
ATG 12-5 & $1 \pm 0.332$ & $0.73 \pm 0.133$ & $0.87 \pm 0.291$ & .72 \\
LAMP-1 & $1 \pm 0.123$ & $0.92 \pm 0.094$ & $0.97 \pm 0.176$ & .89 \\
LAMP-2 & $1 \pm 0.201$ & $0.61 \pm 0.062$ & $0.69 \pm 0.197$ & .18 \\
LC3A-II & $1 \pm 0.149$ & $0.70 \pm 0.106$ & $0.88 \pm 0.165$ & .28 \\
LC3B-II & $1 \pm 0.135$ & $0.66 \pm 0.051$ & $0.86 \pm 0.123$ & .063
\end{tabular}

Protein expression listed as $\mathrm{x}$-fold change \pm standard error of the mean compared with the ACC group. One-way analysis of variance performed to determine the $P$ value. Bonferroni multiple comparison tests were performed. ACC, Regular diet control; HCC, high cholesterol control; HCRV, high cholesterol plus resveratrol; mTOR, mammalian target of rapamycin; p-mTOR, phosphorylated mTOR; $P 70 S 6 K$, p70 S6 kinase; ATG 12-5, autophagy-related gene 12-5 conjugate; $L A M P$, lysosome-associated membrane protein; $L C 3$, light chain 3 . *Significant difference between ACC and HCC groups. and $P=.017$, respectively; Table 1 and Figure 2). The downstream markers of autophagy, including LAMP-2 and ATG 12-5, were also decreased in the HCC group compared with the ACC group $(P=.013$ and $P=.0028$ respectively; Table 1 and Figure 2).

The pigs in the HCRV group had no significant changes in the protein levels of p-mTOR, P70S6K, and LAMP-2 compared with the ACC group (Table 1 and Figure 2). Other markers of autophagy were increased in the ischemic myocardium in the pigs fed a high cholesterol diet, including LC3A-II in the HCC group $(P=.017)$ and beclin 1 in the HCC and HCRV groups $(P=.0083$; Table 1 and Figure 2).

In the nonischemic myocardium, beclin 1 was decreased in the HCC group compared with the ACC group $(P=.0098$; Table 2$)$. Otherwise, no significant changes were found in the nonischemic myocardium among the 3 groups (Table 2).

\section{DISCUSSION}

The results from the present study have demonstrated that in a swine model of metabolic syndrome, resveratrol supplementation partially reverses many of the alterations in autophagy signaling caused by a high cholesterol diet on chronically ischemic myocardium. Previous studies from our laboratory showed resveratrol to modify the risk factors for coronary artery disease in a similar swine model. Pigs fed a high cholesterol diet supplemented with resveratrol had improved global ventricular functioning, increased microvascular relaxation, and reduced markers of inflammation. ${ }^{14,17,20}$ However, the mechanism by which resveratrol exerts these effects remains unclear and likely has several points of regulation, including autophagy.

Autophagy is a highly regulated process that begins with the formation of an "autophagosome." This double membrane structure engulfs cytoplasmic cargo marked for destruction. The outer membrane of the autophagosome fuses with a lysosome, forming an "autolysosome" - where the cargo is ultimately degraded. Several specific proteins markers associated with this process have been well described. Early regulatory proteins, such as mTOR and p70S6K, highlight the relationship of autophagy to other homeostatic processes, because these proteins have been found downstream in insulin signaling and mitogen-associated protein kinase pathways. ${ }^{21,22}$ Other, more specific and essential, markers for autophagy include beclin 1, ATG 12-5, and LAMP. Beclin 1 is essential for autophagy induction. The conjugation of ATG 12 to ATG 5 is required for the initial double membrane formation. LC3 and LAMP are critical downstream markers that are ultimately required to form and stabilize the autolysosome (Figure 3). ${ }^{1,23-26}$ Significant evidence has shown that autophagy is cardioprotective during ischemic stress. ${ }^{1-4}$ 
A

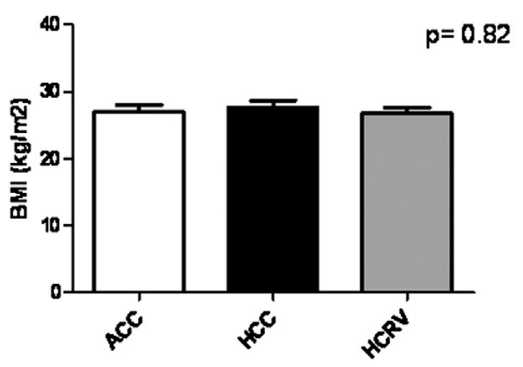

B

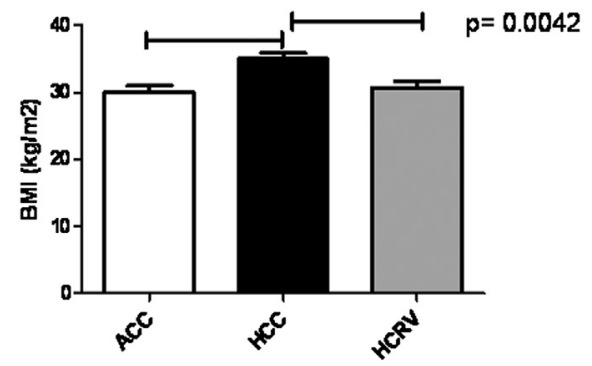

C

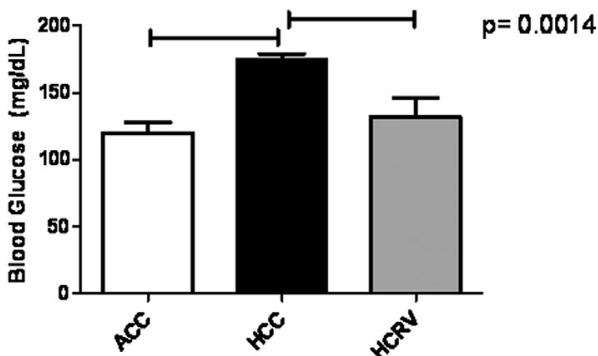

FIGURE 1. Body mass index $(B M I)$ and blood glucose level. A, No difference was found in the BMI between the 2 groups when calculated 4 weeks after initiation of the respective diets. B, At 11 weeks, a significant differences was found in the BMI in the high cholesterol control (HCC) group compared with the regular diet control $(A C C)$ group and the high cholesterol plus resveratrol $(H C R V)$ group $(P=.0042)$. C, The blood glucose level was measured at 11 weeks, 30 minutes after $50 \mathrm{~g}$ of dextrose had been given intravenously. The blood glucose level was significantly greater in the HCC group than in the ACC or HCRV group $(P=.0014)$. One-way analysis of variance was performed to determine the $P$ values. Bonferroni multiple comparison tests were performed.

Although reductions in autophagy can be deleterious in ischemic myocardium, autophagy in excess has also been shown to be harmful. ${ }^{4,25}$ This intricate process is influenced by many factors, and its markers become concentrated and redistributed at different stages. ${ }^{27}$ Thus, a cautious approach must be used when interpreting results involving the markers of autophagy.

The induction of autophagy involves the inhibition of mTOR. This protein is involved in multiple molecular signaling pathways. When phosphorylated, mTOR is activated and becomes an effective downregulator of autophagy. This is known to occur in the setting of caloric excess and hyperlipidemia. ${ }^{22}$ Consistent with this, our results have demonstrated a fourfold increase in p-mTOR in the ischemic myocardium of the high cholesterol-fed pigs compared with those fed a regular diet. However, when the high cholesterol-fed pigs were given resveratrol, the p-mTOR levels were lower, more closely matching the levels in the pigs fed a regular diet (Table 1 and Figure 2). It appears that resveratrol prevents the activation of mTOR seen with a high cholesterol diet, possibly reducing the inhibition of autophagy at this early regulatory step (Figure 3).

A downstream inhibitor of mTOR is p70S6K..$^{21,28}$ Our study has shown that a high cholesterol diet alone significantly decreases the p70S6K levels, and resveratrol supplementation returned the p70S6K levels closer to baseline levels. With the increase in p70S6K resulting from resveratrol supplementation, mTOR activation was likely downregulated, thus promoting autophagy signaling downstream (Figure 3).

In our study, the beclin 1 levels were significantly increased in the ischemic cardiomyocytes of the pigs fed a high cholesterol diet with and without resveratrol. Beclin 1 and its associated complexes regulate the autophagy pathway at multiple stages and in a tissue-specific fashion. These interactions are often transient and unstable and can result in an increase or a decrease in autophagy. Beclin 1-independent autophagy has also been described. ${ }^{24}$ Thus, although it is an important marker of autophagy, the interpretation of beclin 1 levels can be difficult, and elevated levels of beclin 1 will not necessarily indicate increased autophagy. ${ }^{29}$

Similar to beclin 1, resveratrol supplementation does not seem to significantly alter the ATG $12-5$ levels. We found a significant decrease in ATG 12-5 in the high cholesterol-fed pigs with and without resveratrol supplementation compared with the regular diet controls. ATG 12-5 is essential for the expansion and completion of the autophagosome. LAMP-2, also involved in completion of the autophagosome, was significantly decreased only in the high cholesterol-fed pigs that were not given resveratrol $^{27,29}$ (Figure 3).

We found the autophagy marker LC3A-II to be increased threefold in the high cholesterol-fed pigs compared with the pigs fed a regular diet. The high cholesterol-fed pigs 

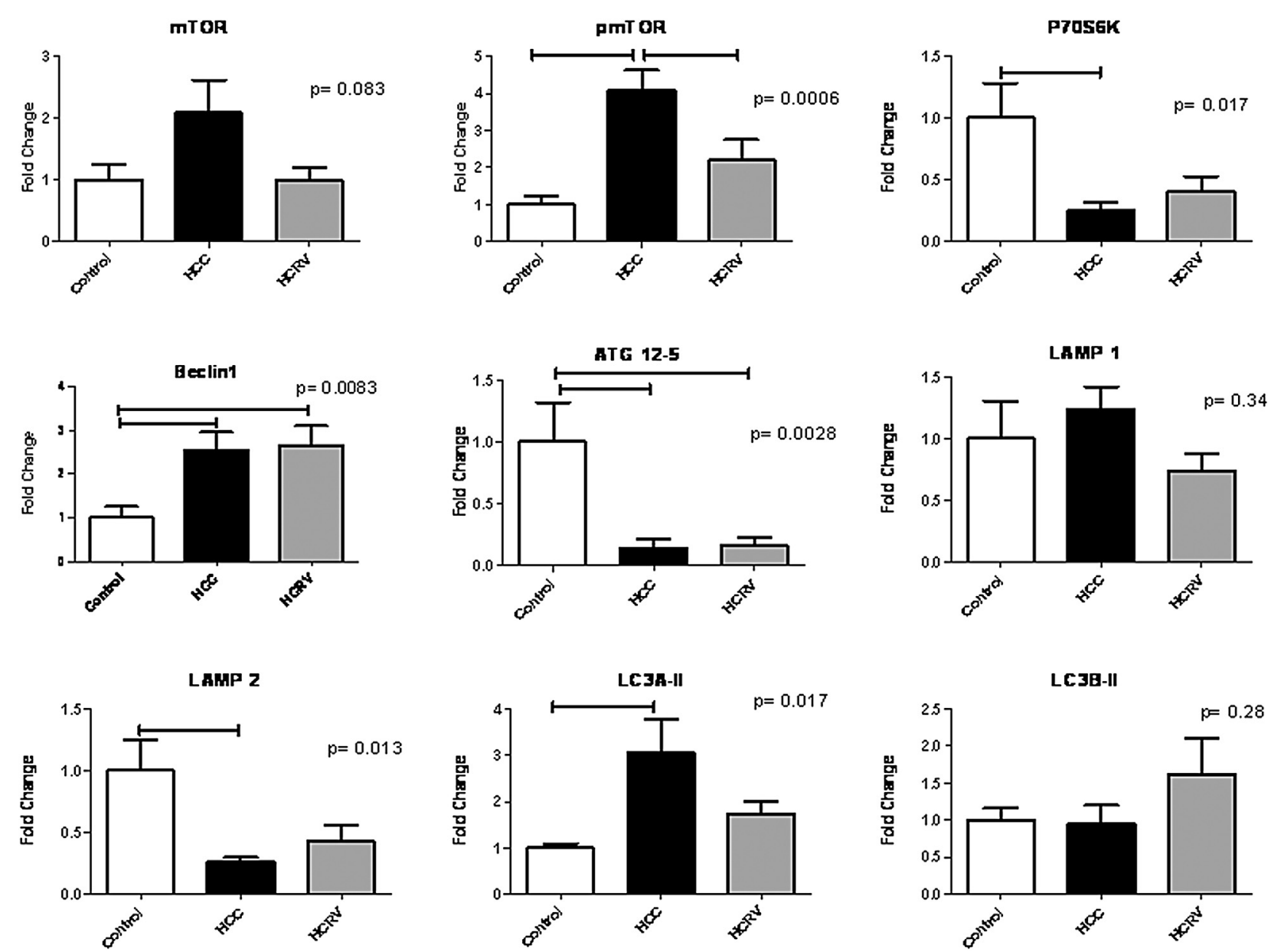

FIGURE 2. Protein expression in ischemic myocardium $x$-fold change \pm standard error of the mean compared with the regular diet control (ACC) group. One-way analysis of variance was performed to determine the $P$ value. Bonferroni multiple comparison tests were performed. $m$ TOR, Mammalian target of rapamycin; pmTOR, phosphorylated mammalian target of rapamycin; P70S6K, p70 S6 kinase; ATG 12-5, autophagy-related gene 12-5 conjugate; $L A M P$, lysosome-associated membrane protein; $L C 3$, light chain 3.

supplemented with resveratrol had LC3A-II levels that were more similar to those of the pigs fed a regular diet. The microtubule-associated protein LC3 is an ubiquitin-like molecule that is a known specific marker for autophagy. LC3-II plays an integral role not only in the formation of the autophagosome, but also in selecting the cytosolic components for degradation ${ }^{1,26,28,29}$ (Figure 3). LC3-II upregulation indicates either an increase in autophagosome formation or a decrease in degradation. No significant changes were found in the LC3A-II levels in the nonischemic myocardium, and no significant changes were found in the LC3B-II levels in the ischemic and nonischemic myocardium.

\section{Study Limitations and Future Directions}

The present study had several limitations. Because we used a large animal model, the number of pigs in each group was kept to a minimum while allowing appropriate power to observe the statistical significance. Thus, the interpretation of the results must be kept within this context. Although the porcine cardiac physiology is similar to that of humans, the pathologic alterations and response to exogenous factors could differ among species. Furthermore, we were unable to comment on the differences in the treatment length or dose-mediated response of resveratrol treatment, because the pigs in the present study received a fixed, relatively high dose of resveratrol. Interpreting the levels of autophagy markers is complicated by their constant flux and multiple points of regulation. Moreover, the timing and measure necessary for autophagy to be cardioprotective remains unclear. Importantly, we did not directly measure autophagy. Instead, as described in similarly designed studies, we used Western blotting to measure the specific and essential markers of the process. The reference standard for directly measuring autophagy is electron microscopy, with which the autolysosome is visualized at different stages in its development. An important criticism of electron microscopy is that it does not allow objective and 


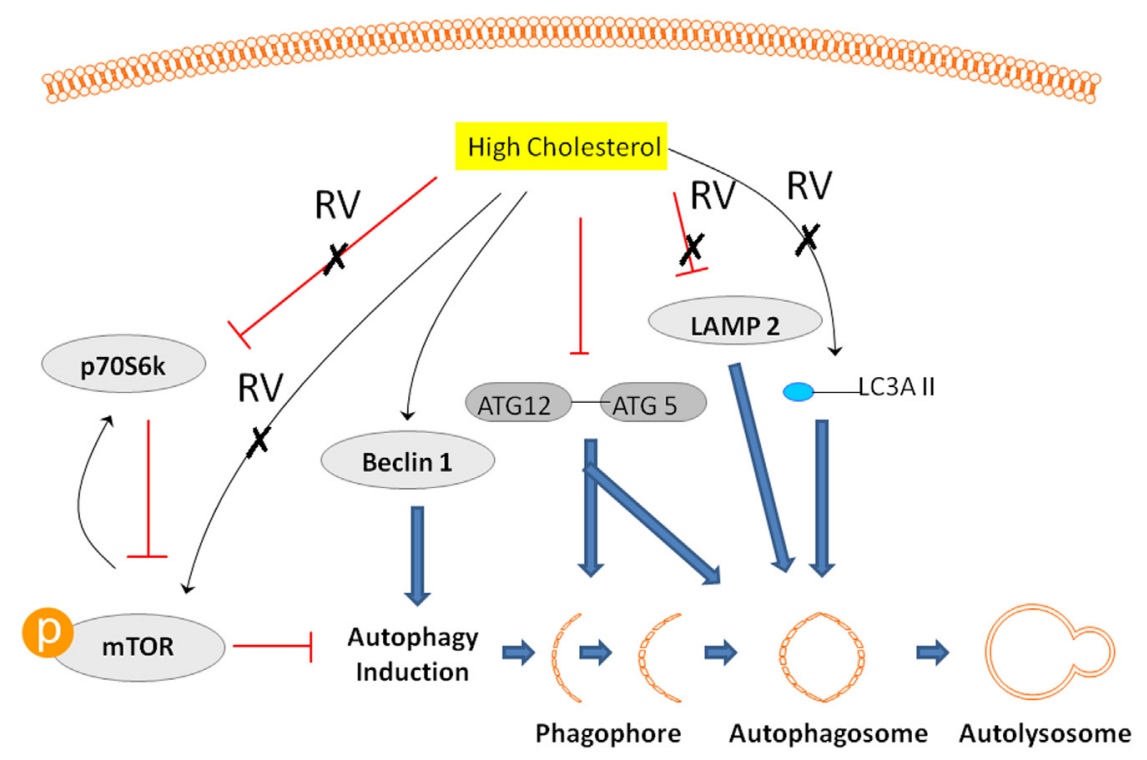

FIGURE 3. Schematic model of the autophagy pathway demonstrating several relevant markers of autophagy that are potentially influenced by a high cholesterol diet and as possible points of regulation by resveratrol $(R V)$. p70S6K, p70 S6 kinase; LAMP, lysosome-associated membrane protein; $A T G$ 12-5, autophagy-related gene 12-5 conjugate; $L C 3$, light chain 3.

reproducible quantification of autophagy, because it is a ubiquitous, homeostatic process. ${ }^{2,4,11,29-31}$ For the purposes of our study, a quantitative measure was necessary; thus, Western blotting was used. Future studies aimed at clarifying the mechanisms relating resveratrol and autophagy are needed. Specifically, the overlap of autophagy and other molecular pathways such as apoptosis should be examined further. Also, because autophagy is a dynamic process, examining it at different points will help elucidate its effects. Although we have emphasized the clinical relevance of a large animal model, attempting to replicate these studies in other species and in cell cultures might help clarify the underlying mechanisms involved.

\section{References}

1. Gottlieb RA, Finley KD, Mentzer RM Jr. Cardioprotection requires taking out the trash. Basic Res Cardiol. 2009;104:169-80.

2. Huang C, Yitzhaki S, Perry CN, Liu W, Giricz Z, Mentzer RM Jr, et al. Autophagy induced by ischemic preconditioning is essential for cardioprotection. J Cardiovasc Transl Res. 2010;3:365-73.

3. Giricz Z, Mentzer RM Jr, Gottlieb RA. Autophagy, myocardial protection, and the metabolic syndrome. J Cardiovasc Pharmacol. 2012;60:125-32.

4. Sala-Mercado JA, Wider J, Undyala VV, Jahania S, Yoo W, Mentzer RM Jr, et al. Profound cardioprotection with chloramphenicol succinate in the swine model of myocardial ischemia-reperfusion injury. Circulation. 2010;122(11 Suppl): S179-84.

5. Hansson GK. Inflammation, atherosclerosis, and coronary artery disease. N Engl J Med. 2005;352:1685-95.

6. Behn A, Ur E. The obesity epidemic and its cardiovascular consequences. Curr Opin Cardiol. 2006;21:353-60.

7. Liu YB, Wu CC, Lee CM, Chen WJ, Wang TD, Chen PS, et al. Dyslipidemia is associated with ventricular tachyarrhythmia in patients with acute ST-segment elevation myocardial infarction. J Formos Med Assoc. 2006;105:17-24.

8. Mehta RH, Bhatt DL, Steg PG, Goto S, Hirsch AT, Liau C-S, et al. Modifiable risk factors control and its relationship with 1 year outcomes after coronary artery bypass surgery: insights from the REACH registry. Eur Heart J. 2008;29: 3052-60.

9. Kassiotis C, Ballal K, Wellnitz K, Vela D, Gong M, Salazar R, et al. Markers of autophagy are downregulated in failing human heart after mechanical unloading. Circulation. 2009;120(11 Suppl):S191-7.

10. Garcia L, Verdejo HE, Kuzmicic J, Zalaquett R, Gonzalez S, Lavandero S, et al Impaired cardiac autophagy in patients developing postoperative atrial fibrillation. J Thorac Cardiovasc Surg. 2012;143:451-9.

11. Jahania SM, Sengstock D, Vaitkevicius P, Andres A, Ito BR, Gottlieb RA, et al Activation of the homeostatic intracellular repair response during cardiac surgery. J Am Coll Surg. 2013;216:719-26; discussion 26-9.

12. Gurusamy N, Lekli I, Mukherjee S, Ray D, Ahsan K, Gherghiceanu M, et al. Cardioprotection by resveratrol: a novel mechanism via autophagy involving the mTORC2 pathway. Cardiovasc Res. 2010;86:103-12.

13. Lekli I, Ray D, Mukherjee S, Gurusamy N, Ahsan MK, Juhasz B, et al. Co-ordinated autophagy with resveratrol and gamma-tocotrienol confers synergetic cardioprotection. J Cell Mol Med. 2010;14:2506-18.

14. Robich MP, Osipov RM, Nezafat R, Feng J, Clements RT, Bianchi C, et al Resveratrol improves myocardial perfusion in a swine model of hypercholesterolemia and chronic myocardial ischemia. Circulation. 2010;122(11 Suppl):S142-9.

15. Chu LM, Lassaletta AD, Robich MP, Sellke FW. Resveratrol in the prevention and treatment of coronary artery disease. Curr Atheroscler Rep. 2011;13:439-46.

16. Robich MP, Chu LM, Chaudray M, Nezafat R, Han Y, Clements RT, et al Anti-angiogenic effect of high-dose resveratrol in a swine model of metabolic syndrome. Surgery. 2010;148:453-62.

17. Robich MP, Osipov RM, Chu LM, Han Y, Feng J, Nezafat R, et al Resveratrol modifies risk factors for coronary artery disease in swine with metabolic syndrome and myocardial ischemia. Eur J Pharmacol. 2011;664:45-53.

18. Burgess TA, Robich MP, Chu LM, Bianchi C, Sellke FW. Improving glucose metabolism with resveratrol in a swine model of metabolic syndrome through alteration of signaling pathways in the liver and skeletal muscle. Arch Surg. 2011;146:556-64.

19. Sabe AA, Elmadhun NY, Robich MP, Dalal RS, Sellke FW. Does resveratrol improve insulin signaling in chronically ischemic myocardium? J Surg Res. 2013;183:531-6.

20. Robich MP, Chu LM, Burgess TA, Feng J, Han Y, Nezafat R, et al. Resveratrol preserves myocardial function and perfusion in remote nonischemic myocardium in a swine model of metabolic syndrome. J Am Coll Surg. 2012;215:681-9.

21. Klionsky DJ, Meijer AJ, Codogno P. Autophagy and p70S6 kinase. Autophagy. 2005;1:59-60; discussion 60-1. 
22. Glazer HP, Osipov RM, Clements RT, Sellke FW, Bianchi C. Hypercholesterolemia is associated with hyperactive cardiac mTORC1 and mTORC2 signaling. Cell Cycle. 2009;8:1738-46.

23. Dong Y, Undyala VV, Gottlieb RA, Mentzer RM Jr, Przyklenk K. Autophagy: definition, molecular machinery, and potential role in myocardial ischemia-reperfusion injury. J Cardiovasc Pharmacol Ther. 2010;15:220-30.

24. Kang R, Zeh HJ, Lotze MT, Tang D. The Beclin 1 network regulates autophagy and apoptosis. Cell Death Differ. 2011;18:571-80.

25. Gottlieb RA, Mentzer RM Jr. Autophagy: an affair of the heart. Heart Fail Rev. 2013;18:575-84.

26. Mizushima N, Yoshimori T. How to interpret LC3 immunoblotting. Autophagy. 2007:3:542-5.

27. Xiao G. Autophagy and NF-kappaB: fight for fate. Cytokine Growth Factor Rev. 2007; 18:233-43.

28. Yang Z, Klionsky DJ. An overview of the molecular mechanism of autophagy. Curr Top Microbiol Immunol. 2009;335:1-32.

29. Barth S, Glick D, MacLeod KF. Autophagy: assays and artifacts. J Pathol. 2010; 221:117-24.

30. Perry CN, Kyoi S, Hariharan N, Takagi H, Sadoshima J, Gottlieb RA. Novel methods for measuring cardiac autophagy in vivo. Methods Enzymol. 2009; 453:325-42.

31. Klionsky DJ, Abdalla FC, Abeliovich H, Abraham RT, Acevedo-Arozena A, Adeli $\mathrm{K}$, et al. Guidelines for the use and interpretation of assays for monitoring autophagy. Autophagy. 2012;8:445-544.

\section{Discussion}

Dr Michael P. Fischbein (Stanford, Calif). Excellent presentation. For my first question, did you look for autophagy directly in your heart specimens rather than just indirectly by measuring the upstream and downstream markers that you showed today?

Dr Sabe. Although we did look at specific essential markers of autophagy, immunoblotting in and of itself is an indirect measure, as you alluded. If we wanted to study this directly, we have to use electron microscopy. That is actually the reference standard for studying autophagy. How that is done is one looks for the autophagosome vacuole in formation at different stages using electron microscopy, and, as one can imagine, that requires quite a bit of experience and expertise. One of the greatest criticisms of this technique is that because autophagy is a ubiquitous process (ie, it occurs in cells all the time), using electron microscopy to study it is not objectively quantifiable, and the specificity might not be ideal. Thus, our method we thought was reproducible, something we could objectively quantify, and was specific.

Dr Fischbein. The second question is biologic proof of concept that autophagy is actually playing a role in your ischemic myocardium. Did you consider blocking autophagy?

Dr Sabe. Because it is an essential process, you cannot completely block it in a large animal model such as this. That being said, other groups have had knockout mice with essential markers "blocked out." Some of the criticism of this has been that with the knockout mice, compensatory mechanisms have occurred that might have confounded the results.

Another technique is actually just diminishing autophagy with medications, such as chloroquine, and that is certainly something that has been done in mouse models and something that we could definitely try. Also, just to clarify, when they did do that in the study in which they used chloroquine, the infarct size was increased and the ventricular remodeling after infarct was worse.
Dr Fischbein. Also, if autophagy is as protective as you are suggesting, did you see an actual decrease in cardiomyocyte apoptosis or necrosis with your treatment?

Dr Sabe. That is a good question. I think the key to that question is "whether autophagy is good?" and "how to prove it by studying apoptosis and necrosis." We began studying apoptosis, and we do have some preliminary data. As I mentioned, the key question is where apoptosis might overlap with autophagy signaling. Some of that data will be coming, and I think it will be interesting.

As far as studying necrosis, we have not done that. That can be done, obviously, with tissue staining. Again, how well or easy that is to quantify would be questionable. However, using magnetic resonance imaging in this animal model and even clinically looking at scar tissue would also be interesting and another future direction to move toward.

Dr Fischbein. Last question is, in all our research, we are always looking for translational potential, and so, how would you predict that we are going to use this drug clinically?

Dr Sabe. That is a great question. At this point, I think more preclinical studies are needed. That is part of the reason we did this in a clinically relevant large animal model, which we think more readily translates to our patient population. That said, resveratrol is available over the counter as an herbal supplement and has been for a long time. Also, resveratrol analogs have been, and are currently being, used safely in clinical trials investigating the potential benefits against cancer and dementia.

I would design a study giving resveratrol for perhaps several weeks before coronary artery bypass grafting in diabetic patients. It would be interesting to study the difference between patients with poorly controlled diabetes and those with well-controlled diabetes. I think you could also do that in a broader scope in a clinical trial, just giving it to patients with angina and diabetes.

Somebody had alluded to this earlier, but at this point I do not see resveratrol being used in its current form. You might need a stronger analog or a more potent analog. That might be because resveratrol is more likely a disease-modifying drug that reduces the risk factors. Also, a number of these patients have already been identified and are receiving insulin and are taking metformin and statins. They might already be receiving the benefits of resveratrol. I think in the future we are going to be using more potent analogs once we have a better understanding of how this works.

Dr Fischbein. Thank you. Nice presentation.

Dr Frank A. Baciewicz, Jr (Detroit, Mich). Are there clinical trials already studying autophagy in the short term just for coronary cases and other cardiac surgery cases? Are you aware of any studies?

Dr Sabe. Just studying autophagy in and of itself, yes. Actually, they were studying autophagy during bypass surgery before and after cardioplegia using atrial tissue sampling. They only considered autophagy in these studies, not with resveratrol, but just specifically studying autophagy and its upregulation in different patients during surgery, before and after cardioplegia. That was recently published.

Dr Baciewicz. What medications are they using?

Dr Sabe. I am not sure whether these patients were getting any additional medications-I do not think they were receiving anything. I think they were specifically just studying atrial 
sampling and measuring autophagy as more of what is occurring during bypass before and after cardioplegia/cardiopulmonary bypass and whether autophagy is a critical process and how autophagy is affected and how that is related to patient outcomes.

Dr Baciewicz. I thought they gave drugs to try to alter the autophagy rate, and they showed some difference.

Dr Sabe. I am thinking of the report by Dr Mentzer. I do not think they did, but I am not sure. Is that what you are referring to?

Dr Baciewicz. Yes.

Dr Sabe. I do not believe they did that, but I would have to review it again. I do not think they did. I do not think they gave any medication. I think they were just studying autophagy and whether it is induced after surgery as more just to determine what happens, and whether it is good or bad.
Dr Y. Joseph Woo (Philadelphia, Pa). Did you prove hypocholesteremia in the latter 2 groups?

Dr Sabe. Yes, we did. That was actually in a previous publication, and we did measure the triglyceride and cholesterol levels.

Dr Woo. Did you also measure the caloric intake?

Dr Sabe. These pigs were given either their regular chow or a fatty diet. The fatty diet was not really a burger and fries, such as on my slides-it was a fat supplemented diet. Also, the pigs would eat everything - that was observed to be sure. The diet was regimented with exactly $2248 \mathrm{kcal}$ for the high cholesterol group.

Dr Woo. Thus, the ingestion of resveratrol did not increase their appetite or their caloric intake?

Dr Sabe. No. They were given a fixed diet. 